\title{
A GENERAL CONVERGENCE CRITERION FOR CONTINUED FRACTIONS $K\left(a_{n} / b_{n}\right)$
}

K. L. HILLAM AND W. J. THRON ${ }^{1}$

1. Introduction. In this article we prove a theorem about convergence of sequences $\left\{S_{n}\right\}$ of linear fractional transformation, generated by a suitably restricted sequence $\left\{s_{n}\right\}$. In the proof we shall make essential use of results recently obtained by Thron [2]. This theorem will then be employed to obtain a general convergence criterion for continued fractions $K_{n=1}^{\infty}\left(a_{n} / b_{n}\right)$. Finally, we shall indicate how certain classical as well as more recent results can be obtained as corollaries.

2. A convergence theorem for sequences of linear fractional : transformations. Consider a sequence $\left\{s_{n}(z)\right\}$, where for each $n \geqq 1$,

$$
s_{n}(z)=\frac{\lambda_{n} z+\mu_{n}}{\rho_{n} z+\sigma_{n}}
$$

is a nonsingular linear fractional transformation. Further, for each $n$, let $s_{n}(z)$ satisfy the mapping property

$$
s_{n}(U) \subset U,
$$

where $U$ is the unit circular region $|z| \leqq 1$. Form from this sequence a new sequence $\left\{S_{n}(z)\right\}$ defined as follows

$$
\begin{aligned}
& S_{1}(z)=s_{1}(z), \text { and for } n>1, \\
& S_{n}(z)=S_{n-1}\left(s_{n}(z)\right) .
\end{aligned}
$$

We see that a sequence of nested circular regions $K_{n}$, with centers $c_{n}$ and radii $r_{n}$, is determined. That is,

$$
K_{n}=S_{n}(U)=S_{n-1}\left(s_{n}(U)\right) \subset S_{n-1}(U)=K_{n-1} .
$$

It is well known that the most general linear fractional transformation which maps the region $U$ onto itself is of the form:

$$
e^{i \omega_{n}} \frac{z+G_{n}}{\bar{G}_{n} z+1}, \text { where }\left|G_{n}\right|=g_{n}<1 .
$$

It follows that we may write

Received by the editors September 12, 1964

1 This research was supported by the United States Air Force under Contract No. AF 49(638)-100. 


$$
S_{n}(z)=c_{n}+r_{n} e^{i \omega_{n}} \frac{z+\bar{G}_{n}}{G_{n} z+1},
$$

where $c_{n}$ is the center and $r_{n}$ is the radius of $K_{n}$. It is then readily established that

$$
S_{n}^{-1}(z)=\frac{\left[c_{n}+\bar{G}_{n} R_{n}\right]-z}{G_{n} z-\left[c_{n} G_{n}+R_{n}\right]}
$$

where for convenience $r_{n} e^{i \omega_{n}}$ has been written as $R_{n}$. From the defining equations (2.3) for $S_{n}(z)$ it is seen that $s_{n}(z)$ man be written as

$$
s_{n}(z)=S_{n-1}^{-1}\left(S_{n}(z)\right) \text {. }
$$

It follows that

$$
s_{n}(z)=\frac{\lambda_{n} z+\mu_{n}}{\rho_{n} z+\sigma_{n}}
$$

may also be written as

(2.7) $s_{n}(z)=\frac{\left[\left(c_{n-1}-c_{n}\right) G_{n}-R_{n}+G_{n} \bar{G}_{n-1} R_{n-1}\right] z+\left[\left(c_{n-1}-c_{n}\right)-\bar{G}_{n} R_{n}+\bar{G}_{n-1} R_{n-1}\right]}{\left[\left(c_{n}-c_{n-1}\right) G_{n} G_{n-1}-G_{n} R_{n-1}+G_{n-1} R_{n}\right] z+\left[\left(c_{n}-c_{n-1}\right) G_{n-1}-R_{n-1}+\bar{G}_{n} G_{n-1} R_{n}\right]} \cdot$

Since there must be equality up to a multiplicative constant between corresponding coefficients in the two expressions for $s_{n}(z)$ it follows that

$$
\frac{\lambda_{n}}{\rho_{n}}=\frac{\left(c_{n-1}-c_{n}\right) G_{n}-R_{n}+G_{n} \bar{G}_{n-1} R_{n-1}}{\left(c_{n}-c_{n-1}\right) G_{n} G_{n-1}-G_{n} R_{n-1}+G_{n-1} R_{n}} .
$$

If $\lambda_{n} / \rho_{n}$ is some constant $k$, then (2.8) reduces to

$$
\left(c_{n-1}-c_{n}\right) G_{n}\left(1+k G_{n+1}\right)-R_{n}\left(1+k G_{n-1}\right)+G_{n} R_{n-1}\left(\bar{G}_{n-1}+k\right)=0,
$$

which can be rearranged to yield

$$
\frac{R_{n}}{G_{n}}=R_{n-1} \frac{k+\bar{G}_{n-1}}{G_{n-1} k+1}+\left(c_{n-1}-c_{n}\right) .
$$

In view of the nestedness of the circular regions $K_{n}$, it follows that

$$
\left|c_{n-1}-c_{n}\right| \leqq\left(r_{n-1}-r_{n}\right) \text {. }
$$

Further, if $|k| \leqq 1$ it is seen from the structure of the expression $\left(k+\bar{G}_{n-1}\right) /\left(G_{n-1} k+1\right)$ that it is not greater than 1 in absolute value. Hence, it follows that 


$$
\left|\frac{R_{n}}{G_{n}}\right|=\frac{r_{n}}{g_{n}} \leqq r_{n-1}+\left(r_{n-1}-r_{n}\right) .
$$

Rearrangement of the expression (2.10) yields a bound for the ratio $r_{n} / r_{n-1}$ of successive radii of the nested circular regions $K_{n}$,

$$
\frac{r_{n}}{r_{n-1}} \leqq \frac{2 g_{n}}{1+g_{n}}=1-\frac{1-g_{n}}{1+g_{n}}=1-\frac{\delta_{n}}{1+g_{n}} \leqq 1-\frac{\delta_{n}}{2},
$$

where $\delta_{n}=1-g_{n}$. Repeated application of (2.11) establishes that

$$
r_{n} \leqq\left[\prod_{p=2}^{n}\left(1-\frac{\delta_{p}}{2}\right)\right] r_{1} \leqq 1 .
$$

Now $\sum_{p=2}^{\infty} \delta_{p} / 2$ and $\prod_{p=2}^{\infty}\left(1-\delta_{p} / 2\right)$ converge or diverge together.

If $\sum_{p=2}^{\infty} \delta_{p} / 2$ diverges, then $\prod_{p=2}^{\infty}\left(1-\delta_{p} / 2\right)$ diverges to zero since its sequence of partial products is monotone decreasing. This establishes that $\lim _{n \rightarrow \infty} r_{n}=0$. In this, the limit point case, it is clear that the sequence $\left\{S_{n}(z)\right\}$ converges at least for all $z$ in the unit circular region $U$ (see $[2$, Theorem 3.1$]$ ).

If $\sum_{p=2}^{\infty} \delta_{p} / 2$ converges, then $\prod_{p=2}^{\infty}\left(1-\delta_{p} / 2\right)$ converges to a limit $R<1$ and different from zero. Thus, $\lim _{n \rightarrow \infty} r_{n}=r \geqq 0$. If $r>0$, this is called the limit circle case. Consider then the quantity

$$
\begin{aligned}
\frac{R_{n}}{G_{n}}-\frac{R_{n-1}}{G_{n-1}} & =R_{n-1} \frac{k+\bar{G}_{n-1}}{G_{n-1} k+1}+\left(c_{n-1}-c_{n}\right)-\frac{R_{n-1}}{G_{n}} \\
& =-\frac{R_{n-1}}{G_{n-1}} \frac{1-g_{n-1}^{2}}{1+k G_{n-1}}+\left(c_{n-1}-c_{n}\right) .
\end{aligned}
$$

Applying (2.13) repeatedly yields

$$
\begin{gathered}
\frac{R_{n+m}}{G_{n+m}}-\frac{R_{n}}{G_{n}}=\left(c_{n}-c_{n+m}\right)-\sum_{p=1}^{m} \frac{R_{n+p-1}}{G_{n+p-1}} \frac{1-g_{n+p-1}^{2}}{1+k G_{n+p-1}} \text { or } \\
\left|\frac{R_{n+m}}{G_{n+m}}-\frac{R_{n}}{G_{n}}\right| \leqq\left(r_{n}-r_{n+m}\right)+\sum_{p=1}^{m} \frac{r_{n+p-1}}{g_{n+p-1}} \frac{\left(1-g_{n+p-1}\right)\left(1+g_{n+p-1}\right)}{\left|1+k G_{n+p-1}\right|} .
\end{gathered}
$$

Since $\sum_{p=2}^{\infty} \delta_{p} / 2$ converges, then $\delta_{p} \rightarrow 0$ and this implies that $g_{n} \rightarrow 1$. Thus, for sufficiently large $n, g_{n+p-1}>1 / 2$. Further recall that $r_{n+p-1}$ $<1$, and $\delta_{n+p-1}=1-g_{n+p-1}$. We now make the additional assumption $|k|<1$. The inequality (2.14) may therefore be rewritten as 


$$
\begin{aligned}
\left|\frac{R_{n+m}}{G_{n+m}}-\frac{R_{n}}{G_{n}}\right| & \leqq\left(r_{n}-r_{n+m}\right)+\sum_{p=1}^{m} \frac{1}{\left(\frac{1}{2}\right)} \frac{\delta_{n+p-1}(1+1)}{1-|k(1)|} \\
& \leqq\left(r_{n}-r_{n+m}\right)+\frac{4}{1-|k|} \sum_{p=1}^{m} \delta_{n+p-1} \\
& =\left(r_{n}-r_{n+m}\right)+\frac{4}{1-|k|} \sum_{q=n}^{n+m-1} \delta_{q \cdot}
\end{aligned}
$$

Since $\lim _{n \rightarrow \infty} r_{n}=r$ and $\sum_{p=1}^{\infty} \delta_{p}$ converges, the sequence

$$
\left\{\frac{R_{n}}{G_{n}}\right\}=\left\{\frac{r_{n} e^{i \omega_{n}}}{g_{n} e^{i \gamma_{n}}}\right\}
$$

converges. This implies that $\left\{\omega_{n}-\gamma_{n}\right\}$ is convergent. The condition that $g_{n} \rightarrow 1$ and $\left\{\omega_{n}-\gamma_{n}\right\}$ converge was shown by Thron [2, Theorem 5.1] to be sufficient to insure that the sequence $\left\{S_{n}(z)\right\}$ converges at least for all $z$ in the interior of the unit circular region $U$.

This completes the proof of the following theorem.

THEOREM 1. If $\left\{S_{n}(z)\right\}$ is a sequence generated by the defining equations (2.3) from a sequence $\left\{s_{n}(z)\right\}$ of nonsingular linear fractional transformations of the form

$$
s_{n}(z)=\frac{\lambda_{n} z+\mu_{n}}{\rho_{n} z+\sigma_{n}},
$$

and if for each $n, s_{n}(z)$ has the property that

$$
s_{n}(U) \subset U,
$$

where $U$ is the circular region determined by $|z| \leqq 1$, and if in addition

$$
\frac{\lambda_{n}}{\rho_{n}}=k \text {, where }|k|<1 \text {, }
$$

then the sequence $\left\{S_{n}(z)\right\}$ converges at least for all $z$ in the interior of $U$.

3. A convergence criterion for continued fractions. For each $n \geqq 1$ let

$$
t_{n}(z)=\frac{a_{n}}{b_{n}+z}, \quad a_{n} \neq 0
$$

and define $T_{1}(z)=t_{1}(z), T_{n}(z)=T_{n-1}\left(t_{n}(z)\right), n \geqq 1$. Then it is well known that $\left\{T_{n}(0)\right\}$ is the sequence of approximants of the continued fraction $K\left(a_{n} / b_{n}\right)$. Let $K$ denote a circular region determined by 


$$
|z-c| \leqq r
$$

where $0 \leqq|c|<r$, so that $0 \in K$. If for each $n$, the elements $a_{n}$ and $b_{n}$ are so restricted that,

$$
t_{n}(K) \subset K,
$$

then by means of Theorem 1 it can be shown that $\left\{T_{n}(0)\right\}$ converges. This is seen as follows.

The transformations

$$
\begin{aligned}
s_{n}(z) & =v^{-1}\left(t_{n}(v(z))\right), & & \text { where } \\
v(z) & =r z+c, & & \text { and } \\
v^{-1}(z) & =\frac{z-c}{r}, & & \text { for all } n \geqq 1,
\end{aligned}
$$

have the property that $s_{n}(U) \subset U$, whenever $t_{n}(K) \subset K$. From (3.1) it is seen that

$$
s_{n}(z)=\frac{(-r c) z+\left(a_{n}-c\left[b_{n}+c\right]\right)}{\left(r^{2}\right) z+\left(r\left[b_{n}+c\right]\right)}
$$

Since $0 \leqq|c|<r$, it is clear that the ratio $\left|\lambda_{n} / \rho_{n}\right|=|-c / r|<1$. Thus, by Theorem 1 , the sequence $\left\{S_{n}(z)\right\}$ defined as in (2.3) converges at least for all $z$ in the interior of $U$. Since further, by repeated application of (3.1),

$$
S_{n}(z)=v^{-1}\left(T_{n}(v(z))\right),
$$

and $v^{-1}(0)$ is in the interior of $U$, it follows that $\left\{T_{n}(0)\right\}$ converges whenever the elements $a_{n}$ and $b_{n}$ are so restricted that

$$
t_{n}(K) \subset K \text {. }
$$

THEOREM 2. Let $K$ be the circular region defined by $|z-c| \leqq r$, where $|c|<r$. Let the elements of the continued fraction $K_{n-1}^{\infty}\left(a_{n} / b_{n}\right)$ satisfy the requirement

$$
t_{n}(K) \subset K, \text { for all } n \geqq 1 \text {, }
$$

where

$$
t_{n}(z)=\frac{a_{n}}{b_{n}+z}, \quad a_{n} \neq 0
$$

then the continued fraction converges to a value $v \in K$.

Since, if $\left|a_{n}\right| \leqq 1 / 4$, the region $K$, defined by $|z-1| \leqq 1 / 2$ is 
mapped into itself by the transformations $a_{n} /(1+z)$, the Worpitzky criterion is a corollary of our theorem, except that our result does not take care of the case where $a_{n}=0$ for some $n$. The same remark applies also to the other two results mentioned below.

Next if $\left|b_{n}\right| \geqq\left|a_{n}\right|+1$ then $t_{n}(U) \subset U$ so that the Pringsheim criterion also can be derived from Theorem 2 .

It was proved by Thron [1] that a sufficient condition for the convergence of a continued fraction of the form $K\left(1 / b_{n}\right)$ is that $\left|b_{n}-2 c\right|$ $\geqq 2\left(c^{2}+1\right)^{1 / 2}$, where $c$ is an arbitrary real number. Since it is known that, if the $b_{n}$ satisfy this condition, then the transformations $1 /\left(b_{n}+z\right)$ map the circle $|z+c| \leqq\left(1+c^{2}\right)^{1 / 2}$ into itself, this result also follows from Theorem 2. The original proof of this theorem was by nonelementary means, that is, essential use was made of the StieltjesVitali theorem. Recently we have given another elementary proof of this result which has the advantage over the present proof that it gives information about the speed of convergence, and shows that the convergence is uniform.

Now consider the restrictions imposed upon the elements $a_{n}$ and $b_{n}$ by the mapping property (3.2). First, it is easily seen that the image $t_{n}(K)$ of $K$ is unbounded unless

$$
\left|b_{n}+c\right|>r \text {. }
$$

Under condition (3.3), $t_{n}(K)$ is determined by

$$
\left|z-\frac{a_{n}\left[b_{n}+c\right]-}{\left|b_{n}+c\right|^{2}-r^{2}}\right| \leqq \frac{\left|a_{n}\right| r}{\left|b_{n}+c\right|^{2}-r^{2}} .
$$

The condition that this circular region be contained in $K$ is expressed by

$$
\left|\frac{a_{n}\left[b_{n}+c\right]-}{\left|b_{n}+c\right|^{2}-r^{2}}-c\right|+\frac{\left|a_{n}\right| r}{\left|b_{n}+c\right|^{2}-r^{2}} \leqq r .
$$

This may be rewritten as

$$
\left|a_{n}\left[b_{n}+c\right]^{-}-c\left(\left|b_{n}+c\right|^{2}-r^{2}\right)\right|+\left|a_{n}\right| r \leqq r\left(\left|b_{n}+c\right|^{2}+r^{2}\right)
$$

Now if

$$
\begin{aligned}
\left|a_{n}\right|\left|b_{n}+c\right|+|c|\left(\left|b_{n}+c\right|^{2}-r^{2}\right)+ & \left|a_{n}\right| r \\
& \leqq r\left(\left|b_{n}+c\right|^{2}-r^{2}\right)
\end{aligned}
$$

then the inequality (3.4) is clearly satisfied. Rearranging and simplifying (3.5) yields

$$
\left|a_{n}\right| \leqq(r-|c|)\left(\left|b_{n}+c\right|-r\right) .
$$


This completes the proof of another corollary of Theorem 4.1.

CoRollary 1. Let $r$ be a positive number and let $c$ be a complex number $|c|<r$. Then the continued fraction $K_{n=1}^{\infty}\left(a_{n} / b_{n}\right)$ converges if for all $n \geqq 1$

$$
0<\left|a_{n}\right| \leqq(r-|c|)\left(\left|b_{n}+c\right|-r\right) .
$$

\section{REFERENCES}

1. W. J. Thron, Twin convergence regions for continued fractions $b_{0}+K\left(1 / b_{n}\right)$. II, Amer. J. Math. 71 (1949), 112-120.

2. - Convergence of sequences of linear fractional transformations and of continued fractions, J. Indian Math. Soc. 27 (1963), 103-127.

BrighaM Young UNTVERSITY AND

UNIVERSITY OF COLORADO 